\title{
29 Die krimtatarische Bevölkerung nach dem Krimkrieg
}

\begin{abstract}
Von den hochrangigen Krim-Adligen hat nicht einer von ihnen seine Kinder in die [krimtatarische] Schulabteilung gegeben, obwohl diese 28 Jahre lang bestand. Viele Beamte und Wahlhelfer schrieben und schreiben nur mit Mühe ihren Vor- und Nachnamen auf Russisch, immer haben sie es vermieden, unter irgendeinem Vorwand, ihre Kinder gründlich Russisch lernen zu lassen. Die Folge war, dass früher, als die Krimbevölkerung mehrheitlich noch aus Tataren bestand, die tatarische Schulabteilung keinerlei Nutzen brachte, dass kaum die Notwendigkeit gegeben war, diese für die Tataren aufrechtzuerhalten. [...] Niemand hat jemals den tatarischen Adel daran gehindert, ihre Kinder in gemeinsame [gemischtnationale, K.S.J.] Lehranstalten zu geben. Im Gegenteil, alle örtlichen Kräfte haben sie, wann immer sie konnten, dazu ermuntert, aber ohne jeden Nutzen, weil sie sich augenscheinlich weigerten, ihre Kinder gemeinsam mit anderen Nationen zu erziehen, anscheinend fürchten sie, dass ihre Kinder, wenn sie eine Ausbildung erhalten, aufhören, Muselmanen [,,byt' Musul'manami“"] zu sein [...] Das ist keine Verleumdung, sondern die reine Wahrheit. ${ }^{1}$
\end{abstract}

So düster schätzte der ungenannte Autor 1863 in der „Zeitschrift des Ministeriums für Volksbildung“ das Interesse der krimtatarischen Eliten an der Bildung ihres Nachwuchses ein. Diese hielten, wie es weiter hieß, ebenso wie die Krim-Karäer, „ihre Kinder in tiefer Dummheit“, während die rabbinischen Juden bildungsbeflissen seien. ${ }^{2}$ Der Autor lieferte eine detaillierte Beschreibung des Zustandes des Simferopol'er Bildungswesens, des Zentrums des Taurischen Gouvernements, in den ersten Jahren nach dem Krimkrieg. Darin sparte er auch nicht an Kritik an den staatlichen Stellen, welche insbesondere die Mädchenbildung vernachlässigen würden. Die gegen Ende der 1850er Jahre einsetzende große Emigrationswelle der TatarInnen thematisierte er ebenfalls: Bis zur „Aussiedlung“ („do vyselenija“) der krimtatarischen Bevölkerung hätten diese ein Drittel der Bevölkerung gestellt, 1863 jedoch nur noch ein Elftel; ob er dies als Verlust empfand oder nicht, ist seinen Zeilen nicht zu entnehmen. ${ }^{3}$

Die krimtatarische Gemeinschaft befand sich nach dem Krimkrieg in einer grundlegenden Krise: Das ihnen kollektiv von Teilen der zarischen Administration und slavischen UntertanInnen entgegengebrachte Misstrauen sowie die prekäre wirtschaftliche Lage hatte viele - wie geschildert - migrieren lassen. Kulturell und gesellschaftlich, so zumindest das Urteil des Kenners der krimtatarischen Geschichte, Edward Lazzerini, war ihre Lage zusätzlich durch Passivität

1 Vesti (1863), 242f.

2 Vesti (1863), 245.

3 Vesti (1863), 242.

Ә OpenAccess. (C) 2020 Kerstin S. Jobst, publiziert von De Gruyter. (cc))BY Dieses Werk ist lizenziert unter der Creative Commons Attribution 4.0 International. https://doi.org/10.1515/9783110520620-031 
auf intellektuellem und künstlerischem Gebiet gekennzeichnet, worauf die oben erwähnte Verweigerung des Besuchs staatlicher Schulen hindeutet. ${ }^{4}$ Es war allerdings keineswegs so, dass die Krim-MuslimInnen die Schule an sich verweigerten, denn es gab ein flächendeckendes Netz tatarischer Bildungseinrichtungen, welches auch in Anspruch genommen wurde. Die Bildungseinrichtungen standen allerdings unter der Ägide der muslimischen Geistlichkeit. Dieser oblagen überdies das Führen von Geburts- und Sterberegistern und die Rechtsprechung in Angelegenheiten, welche die muslimische Gemeinschaft betrafen. ${ }^{5}$ Ihrerseits bestand kein Interesse, dass sich daran etwas änderte. In den Grund- und höheren Schulen - den mekteb bzw. medrese - blieben somit religiöse Inhalte und das Studium des Arabischen und des Korans auch im 19. Jahrhundert vorherrschend. Krimtatarisch in Wort und Schrift wurde dort nicht gelehrt, und die Versuche der imperialen Administration zwischen den 1840er und 1860er Jahren zur Einführung eines Basisunterrichts in russischer Sprache war ohne rechte Energie durchgeführt worden und hatte somit wenig Erfolg gezeitigt. ${ }^{6}$ Hinzu kam der nicht überzeugende Zustand des gesamtrussländischen Bildungswesens, das nicht wirklich eine Alternative zu den muslimischen Einrichtungen bot. Es erhielt erst in der Reformära die entscheidenden Impulse, die auch unter der krimtatarischen Bevölkerung die AbsolventInnenzahlen moderat ansteigen ließen. Die sog. Russisch-Tatarischen Lehranstalten wurden zum großen Teil vom Erziehungsministerium finanziert und sollten die „Russifizierung und Verschmelzung [,slijanie“] aller innerhalb der Grenzen unserer Heimat lebenden Fremdstämmigen [,inorodcy“] mit den Russen befördern“, wie es in einer Direktive dieser Stelle hieß. ${ }^{7}$ Die Vermutung unseres oben genannten Autors, dass die muslimische Elite aus Angst vor der Entfremdung bislang praktizierter religiöser und lebensweltlicher Regeln ihrem Nachwuchs den Besuch dieser Einrichtungen verweigerte, war nicht aus der Luft gegriffen, war diese ,Entfremdung doch erklärtes Ziel der zarischen Verantwortlichen. Die Bewahrung ihrer muslimisch-tatarischen Identität inmitten einer zunehmend slavisch-orthodoxen Umgebung erschien krimtatarischen AkteurInnen gerade im Bewusstsein ihrer seit den 1860er Jahren so stark dezimierten Zahl wichtig; nicht wenige wählten daher den Weg der Abgrenzung von der slavischen Mehrheitsgesellschaft. Auch die Emigration war ein Versuch, die durch den Modernisierungsdruck gefährdete muslimische Identitätskonstruktion $\mathrm{zu}$ stabilisieren. Eine weitere Auswanderungswelle, nachdem 1874 im Zarenreich die allgemeine Wehrpflicht eingeführt worden war, unter-

\footnotetext{
4 Lazzerini (1988), 136.

5 Fisher (1978), $96 \mathrm{f}$.

6 Kirimli S. (1990), 29-39.

7 Zitiert nach Kirimli S. (1990), 39.
} 
streicht dies.Viele Krimtataren fürchteten, in der zarischen Armee ihren religiösen Geboten nicht nachgehen zu können. ${ }^{8}$ Zugleich erfreuten sich krimtatarische Soldaten eines hohen Renommees. ${ }^{9}$ Selbst nach dem Krimkrieg, in dem nach verbreiteter Auffassung die tatarische Bevölkerung mit den Gegnern Russlands kollaboriert hätte, zeigte sich Alexander II. von deren militärischen Leistungen beeindruckt: Im Jahre 1863 wurde als Anerkennung für deren Verdienste eine spezielle berittene Einheit geschaffen, welche dem Zaren persönlich unterstellt wurde. ${ }^{10}$

Ein komplexes Wechselspiel zwischen Integration und Abschottung, zwischen Vertrauen und Misstrauen war im krimtatarisch-russischen Kontakt konstituierend. So soll noch angeführt werden, dass auch akkulturierende Erscheinungen und ein wechselseitiger Austausch zwischen alten und neuen KrimbewohnerInnen existierten - auch wenn es kaum gemischtreligiöse Heiraten gab. ${ }^{11}$

Russische AutorInnen waren überzeugt, dass die ,rückständigen‘ KrimtatarInnen nur mittels der Ausbildung in imperialen Institutionen und in russischer Sprache zivilisiert und damit ,vollwertig‘ werden könnten. Dabei wurde in der Regel die Freiwilligkeit gegenüber Zwangsmaßnahmen bevorzugt und das Versagen der zarischen Verantwortlichen beklagt, die zu wenig Anreiz für den Besuch russischsprachiger Schulen boten. ${ }^{12}$ Interessant ist auch, dass den traditionellen krimtatarischen Bildungseinrichtungen zuweilen ein recht gutes Zeugnis ausgestellt wurde: Zu Beginn der 1880er Jahre, so hieß es in einer Analyse zur Lage der krimtatarischen Bevölkerung in der Nachemigrationsphase, könnten immerhin siebzig Prozent beiderlei Geschlechts (!) lesen und schreiben, aber eben nicht auf Russisch und nur selten auf Krimtatarisch. ${ }^{13}$ Auch wenn diese Zahl an sich nichts über die Qualität dieser Fähigkeit aussagt, erscheint sie im Vergleich mit anderen (auch russischsprachigen) Teilen des Imperiums hoch. ${ }^{14}$ Dass in den von der Geistlichkeit beaufsichtigten Schulen auf der Krim allerdings die religiöse Unterweisung absolute Priorität hatte, war nicht wegzudiskutieren.

Seitens krimtatarischer Akteure wurde dies genauso kritisiert wie die allgemein pessimistische Grundstimmung in der muslimischen Bevölkerung in den

8 Vgl. dazu Davies F. (2013), $165 \mathrm{f}$.

9 So z. B. der General-Gouverneur Neurusslands und Vertrauter der Zarin Katharina II, Platon A. Zubov (1767-1822). Vgl. Kirpenko (1897).

10 Vgl. Fisher (1978), 88, sowie Zenkovsky (1960), $124 \mathrm{f}$.

11 Jobst (2010).

12 Vgl. Jobst (2007b), 202-204.

13 Gol'denberg (1883), $84 \mathrm{f}$.

14 Zur Geschichte der Alphabetisierung im Russländischen Reich vgl. Brooks (1985). 
Jahrzehnten nach dem Krimkrieg. Zu Beginn der 1880er Jahre entstand schließlich auf der Krim selbst (ähnlich wie bereits vordem bei den TatarInnen der Wolgaregion) eine Bewegung ${ }^{15}$, die sowohl für diese selbst als auch für die Ausbildung einer spezifischen muslimisch-russischen Identität von fundamentaler Bedeutung werden sollte, nämlich der sog. Djadidismus (in etwa „neue Methode“; arab.: al-usul al gadida; pers.: törki usul-i gadid). Als „the architect of modernism among Muslim Turkic subjects of the Russian Empire“ gilt İsmail Gaspiralı (krimtat.; russ. Ismail Gasprinskij; 1851-1914). ${ }^{16}$ Er gehörte zu der ersten Generation der „Trans Imperial People“ (James H. Meyer) der Russlandmuslime, zu denen auch der Wolgatatare Yosıf Aqçura (tat.; russ. Jusuf Akčurin; 1876-1935) oder der Aseri Әli bəy Hüseynzadə (aser.; russ. Ali-bek Gusejnzade;1864-1940) zählen, und welche Verbindungen zu Intellektuellen im Osmanischen Reich unterhielten. ${ }^{17}$ Wie Aqçura oder כli bəy Hüseynzadə erhielt Gaspıralı sowohl eine Ausbildung im Zarenreich selbst (in Moskau) und im westlichen Europa (Paris) als auch in Istanbul. ${ }^{18}$ Seine Rolle bei der Schaffung eines muslimischen Kommunikationsraumes innerhalb des Russländischen Reiches wird von der Forschung immer wieder unterstrichen. ${ }^{19}$ Zugleich sah er sich, worauf Ulrich Hofmeister erst kürzlich hingewiesen hat, als Muslim von der Halbinsel Krim offenbar in einer „Mittlerposition“ zwischen dem europäischen Russland und dessen muslimisch bewohnten Gebieten in Zentralasien. Dazu gehörte, dass er die Rolle der Bildung als entscheidenden Faktor für die sog. Zivilisierung der muslimischen UntertanInnen identifizierte und den Islam als solches - und anders als viele seiner russischen ZeitgenossInnen - nicht grundsätzlich als Modernisierungshindernis sah. ${ }^{20}$ Im Jahre 1881 erschien seine Schrift „Russkoe Musul'manstvo“ („Der russische Islam“) ${ }^{21}$ und ab 1883 die Zeitschrift „Terciman/Perevodčik“ („Der Übersetzer“), die seine Auffassungen weit über eine krimtatarische LeserInnenschaft hinaus popularisierte. ${ }^{22}$ Zentrale Forderung war die Erneuerung des Islams im Zarenreich durch die Übernahme des westlichen, konkret des russischen Bildungsmodells. Das religiöse Erziehungsmonopol der Geistlichkeit sollte aufgehoben werden, die Verständigung der RusslandmuslimInnen durch den Gebrauch

15 Dazu im Detail Noack (2000).

16 Lazzerini (1997), 177.

17 Meyer J. (2014), 21.

18 Zur Biographie Gaspiralıs vgl. Lazzerini (1973).

19 Vgl. zuletzt Tuna (2015).

20 Hofmeister (2017), besonders 124 und 135.

21 Erste Ausgabe: Gasprinskij (1881).

22 AbonnentInnen hatte der „Terciman“ auch im Osmanischen Reich und selbst in Indien, vgl. Fisher (1978), 103. 
einer von ihm entwickelten Turk-Standardsprache, die (vereinfacht ausgedrückt) eine Variante des gesprochenen Tatarisch ohne arabische und persische Wörter war, gefördert werden. In dieser war auch der turksprachige Teil des zweisprachigen, auch russische Artikel enthaltenden „Terciman“ geschrieben. ${ }^{23}$ Zur Verbreitung dieser Sprachvariante entwickelte er eine neue, effektive Lehrmethode. Im Jahre 1884 wurde auf seine Initiative hin in Bachčisaraj, wo er vordem einige Jahre als Bürgermeister tätig gewesen war, eine erste Reformschule gegründet, in der das Lesen und Schreiben nach dieser Methode genauso gelehrt wurde wie eine weltliche Bildung sowie die russische Sprache. Anfangs zeigten sich MuslimInnen in anderen Teilen des Imperiums wenig beeindruckt von Gaspiralıs Engagement, schließlich übernahmen jedoch beispielsweise WolgatatarInnen dieses Modell. ${ }^{24}$ Gaspiralı und seine Mitstreiter und Mitstreiterinnen - die gleichberechtigte Teilnahme von Frau und Mann am gesellschaftlichen Leben wurde eine zentrale Forderung der Djadidisten ${ }^{25}$ - waren überzeugt, dass nur eine moderne, weltlich geprägte muslimische Gesellschaft sich dauerhaft der Russifizierung entziehen könnte.

Letztlich formierte sich von der Krim ausgehend eine auf die ,gemeinsame Erfahrung، der turkstämmigen Abstammung, auf eine gemeinsame Sprach- und Kulturgemeinschaft einschließlich der Religion und auf den Rekurs auf eine ,gemeinsame Geschichte‘ (die der Goldenen Horde) gestützte ,moderne‘ Gruppenidentität. Der „djadidistische [...] Diskurs schuf [...] mithin eine Gemeinschaft muslimischer Eliten aus allen Teilen Russlands als Kommunikationsverbund“, so das Urteil Christian Noacks. ${ }^{26}$ Gaspiralı und seine Generation mögen den Djadidismus noch als eine primär kulturelle Bewegung interpretiert und geplant haben, letztlich war ihr Tun allerdings politisch. ${ }^{27}$ Dennoch: Was bedeutete ein ,weltlicher Islam' für die gedachte Gemeinschaft aller Muslim, die Umma, und für die Rolle des Osmanischen Reiches? War der Djadidismus eine Art Nationalbewegung der KrimtatarInnen oder der RusslandmuslimInnen insgesamt? Wie wurde das Verhältnis zur ethnisch türkischen Bevölkerung im Osmanischen Reich interpretiert? Welche Folgen erwuchsen dadurch für St. Petersburg oder Istanbul? Gaspiralı selbst betonte zwar immer wieder - bei dezidierter Ablehnung einer

23 Vgl. Bennigsen u. Lemercier-Quelquejay (1964), 35-46.

24 Noack (2000), $147 \mathrm{f}$.

25 Seine Tochter Şefiqa Gaspıralı (krimtat.; russ. Šefika Gasprinskaja; 1886-1975) gehörte zu seinen engsten MitarbeiterInnen und war Herausgeberin mehrerer für eine weibliche Leserschaft konzipierter Zeitschriften. In westlichen und slavischen Sprachen fehlt leider eine zufriedenstellende Untersuchung, vgl. aber Gankevič (1994); Hablemitoğlu u. Hablemitoğlu (1998).

26 Noack (2000), 150.

27 Vgl. z. B. Gankevič u. Šendrikova (2008). 


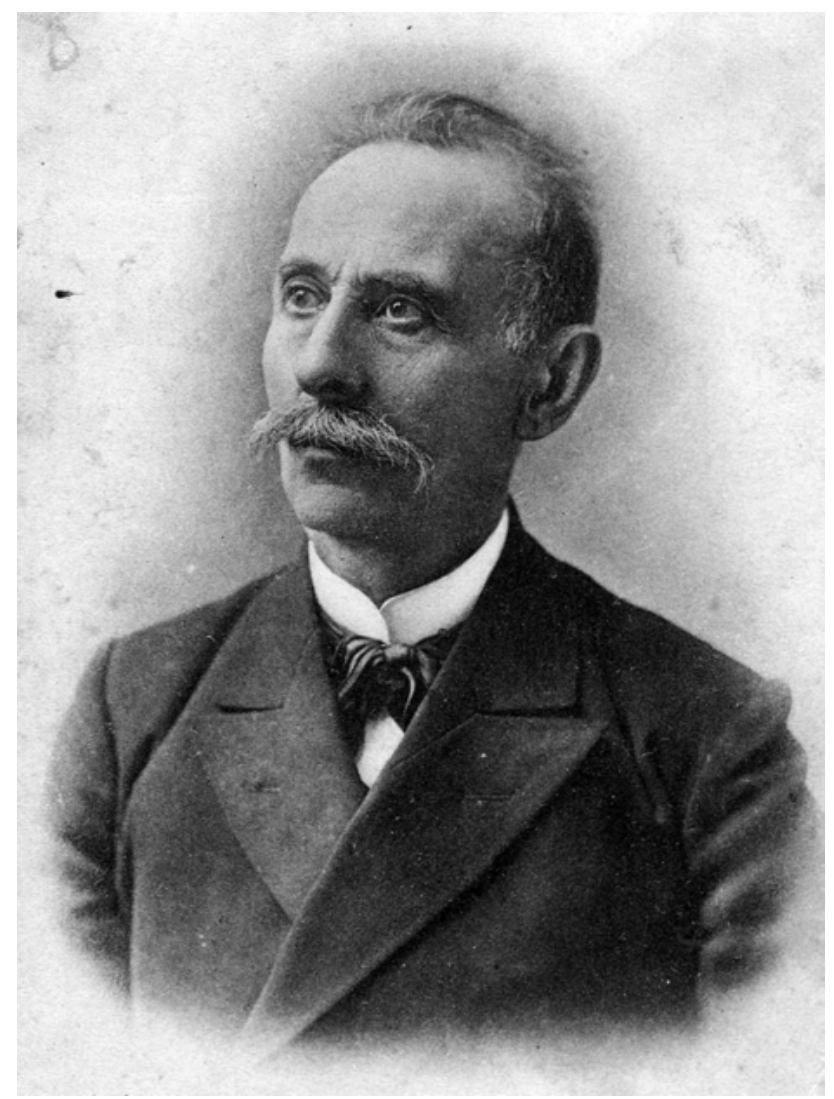

Abb. 10: İsmail Gaspıralı (Ismail Gasprinskij)

russifizierenden Politik - die Verbundenheit der RusslandmuslimInnen mit dem Imperium, ihm schlug aber gerade seitens nationaler und orthodoxer russischer Eliten, die in jedem Muslim einen religiösen Fanatiker sehen wollten, immer wieder ein kalter Wind entgegen. ${ }^{28}$ Nach der Russischen Revolution von 1905 zeigte sich, dass der Djadidismus auch innerhalb der muslimischen Gemeinschaft kritisiert wurde: Seitens der sich zunehmend national als tatarisch oder ethnischtürkisch definierenden sog. Jungtatarischen Bewegung ${ }^{29}$ regte sich ebenso Widerstand wie seitens der traditionellen muslimischen Geistlichkeit, die von russischen Stellen weiterhin bevorzugt wurde. Am Beispiel der von Gaspiralı ge-

28 Hofmeister (2017), 124-128.

29 Kirimli H. (1993), hier 534. 
prägten Bewegung lässt sich Folgendes zeigen: Zum einen, dass auch die vermeintlich so rückständige krimtatarische Bevölkerung in etwa parallel zu anderen Nationalitäten innerhalb der zeitgenössischen Imperien am Beginn eines Nationsbildungsprozesses stand, der spätestens in den Jahren vor dem Ersten Weltkrieg große Teile der intellektuellen Eliten berührte. ${ }^{30}$ Es begann eine Entwicklung, in der immer mehr KrimtatarInnen die Halbinsel als geistig-emotionalen Bezugspunkt sahen und nicht mehr länger das Osmanische Reich bzw. die abstrakte Umma. ${ }^{31}$ Zum anderen werden die existierenden Handlungsspielräume der krimtatarischen Bevölkerung bzw. ihrer Eliten sichtbar. Erst durch die imperiumsweiten Reformen konnten diese etwa in der lokalen Selbstverwaltung genutzt werden - und sie wurden genutzt, was u.a. die Installierung der Gaspiralıschen Reformschulen belegt. Dennoch: „,[D]en krimtatarischen Eliten [war] unbestritten der Primat des selbständigen politischen Handelns entzogen und die eigenverantwortliche gesellschaftliche Gestaltungskompetenz zumindest eingeschränkt, so wie es in kolonialen Kontexten üblich ist. “32 Und dass das ehemalige Krim-Chanat eine Kolonie und dessen Titularnation eine unterworfene Nationalität war ${ }^{33}$, daran kann kein Zweifel bestehen. Kolonialismus gibt es allerdings in unterschiedlichen Varianten und Ausprägungen. Im Unterschied beispielsweise zu einigen zentralasiatischen Eroberungen des Russländischen Reiches war die Krim - wie beschrieben - fest in den Reichsverband eingebunden, die für die zentralrussischen Gebiete eingeführten rechtlichen Normsetzungen galten im Wesentlichen auch dort. Die krimtatarische Bevölkerung unterlag auch nicht dem 1822 eingeführten „Statut über die Verwaltung der Fremdstämmigen“ („Ustav ob upravlenii inorodcev"), die als eine besondere Rechtskategorie für eine große Zahl nicht-slavischer BewohnerInnen des Zarenreichs geschaffen worden war und die somit außerhalb des Rechtskodex der zentralrussischen Gebiete stand. Gleichwohl wurden KrimtatarInnen häufig abwertend als „inorodcy“ (Fremdstämmige)

30 So auch Kappeler (1993), 196.

31 Williams (2001), 190.

32 Jobst (2017a), 101.

33 Nach dem freilich zur damaligen Zeit noch nicht ausgebildeten Völkerrecht hat die krimtatarische Bevölkerung als sog. indigene bzw. autochthone Nationalität zu gelten. Vgl. United Nations (2007). Vgl. auch United Nations (2013), 6: „Indigenous communities, peoples and nations are those which, having a historical continuity with pre-invasion and pre-colonial societies that developed on their territories, consider themselves distinct from other sectors of the societies now prevailing on those territories, or parts of them. They form at present non-dominant sectors of society and are determined to preserve, develop and transmit to future generations their ancestral territories, and their ethnic identity, as the basis of their continued existence as peoples, in accordance with their own cultural patterns, social institutions and legal system.“ 
bezeichnet, auch wenn dieser Begriff ursprünglich nicht pejorativ gewesen war. ${ }^{34}$ Und dieses Spannungsfeld, welches übrigens auch in sowjetischer Zeit bestand (Kapitel 32), war und ist kennzeichnend für das Verhältnis zwischen TatarInnen und der russischen Macht auf der Halbinsel: Auch in zarischer Zeit gab es keine Benachteiligung auf der juristischen Ebene. Mindere Rechte auf Grund der Nationalität (auf Grund der sozialen Zugehörigkeit allerdings schon) existierten nicht, strukturelle und praktizierte Diskriminierungen allerdings sehr wohl. Wie allerdings u.a. das Beispiel der Djadidisten zeigt, verstanden Akteure die sich bietenden Partizipationsmöglichkeiten zu nutzen, besonders nach der Revolution von 1905.

34 Slocum (1998). 